\title{
Foreword for Visualized Cancer Medicine: The era for dynamic visuals is here
}

\author{
Chao-Nan Qian ${ }^{1}$, Francesco Pezzella ${ }^{2}$, and Zhimin $\mathrm{Lu}^{3}$ \\ 1 Guangzhou Concord Cancer Center, Dongfeng Road, 510045 Guangzhou, PR China \\ 2 Nuffield Department of Clinical Laboratory Sciences, University of Oxford, John Radcliffe Hospital, Oxford, UK \\ ${ }^{3}$ Cancer Center and Institute of Translational Medicine, Zhejiang University, 268 Kaixuan Road, Hangzhou, PR China
}

\begin{abstract}
We have seen in many circumstances of cancer research and clinical practice that the process itself is more critical and valuable than the result. For better presenting the natural movements of studied subject as well as the processes of medical intervention in treating patient, we proudly launch Visualized Cancer Medicine as a peer-reviewed publication platform covering all relevant topics in which videos play a critical role for presenting the results or the procedures. We appreciate the constant supports from our rigorous authors, dedicating editorial staff members, creative informative technology engineers, and enthusiastic readers. We hope that our small step of establishing Visualized Cancer Medicine for better scientific presentation would foster giant leaps of our understanding on cancer, which would subsequently benefit human being in many ways.
\end{abstract}

Key words: Publication, Cancer, Video, Treatment, Research, Oncology.

Imaging has always been a powerful information tool. The first inherited documents left by human ancestors were images. As one of the great languages of the ancient world, Egyptians had a written language based on pictures (Figure 1A) while two other great scripture systems, the Chinese ideogram (Figure 1B) and the Mesopotamia cuneiform, were originally transformed from pictorial writing systems.

In the absence of movies and of anything equivalent to newspapers, with just a few people able to read a limited number of publications, images were used to provide news and information in ancient times. Two famous examples are the column built in Rome by Emperor Traianus and the Bayeux Tapestry made in England in the 1070s. On the column, the story of the conquest of the Kingdom of Dacia by the Romans is narrated (Figure 2). Centuries later, the $70 \mathrm{~m}$ long Bayeux Tapestry was weaved, depicting the events leading up to the Norman conquest of England. Now, we are experiencing a revival of "pictorial writing" in our daily life as computer and other electronic screens display a huge variety of pictures, symbols, and videos that have changed and continue to profoundly impact our lives (Figure 3).

Medicine and biology are no exceptions. As we strive for better health and medicine, new approaches in the fields of cancer prevention, therapeutic management, and rehabilitation are accelerating, with rapidly accumulating data and evidence. Inevitably, the value of imaging and in particular the "moving imaging" is coming back. There are many advantages in using videos to present research findings, medical procedures, and complicated theories. The dynamic relationship of multiple elements, especially those dependent on spatial arrangement can be accurately presented. Moreover, human beings, as mammals, are more sensitive to moving targets than static objects. The impression of a good movie lasts far longer than a motionless picture. Not to mention, that some medical procedures are almost indescribable and movies are the only appropriate mode of presentation.

In publications, the conventional way of presenting research findings using tables, figures, and literal descriptions does not always sufficiently and accurately reflect the facts, especially, when a dynamic procedure is itself the key element of the findings. Videos have been used frequently in journals as supplementary materials for research findings in recent years. However, this information is not reader-friendly as readers have to dig around in the supplementary documents that are separated from the main text. Furthermore, in many cases, the moving image itself confers the central message of the paper and should be in the main text. The videos in the field of oncology are now part of both the daily clinical work and the researcher's activities. One of the earliest applications is in surgery; watching a surgical procedure as a movie rather than glimpsing some pictures can be extremely useful. In clinical imaging development, videos have helped to foster great advancements in pedagogy. Ultrasound provides the possibility to record the movement of organs, especially heart. Three dimensional reconstructions of radiological imaging can be explored using virtual reality. Microscopy also benefits from the moving image techniques like the intravital multiphoton micrograph through surgically implanted windows in experimental animals. 
A

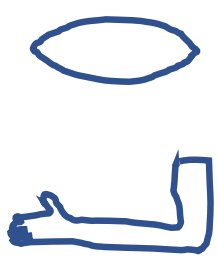

B

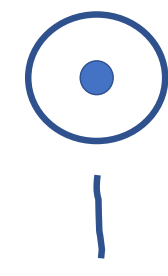

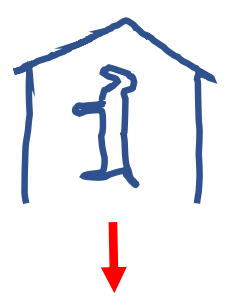

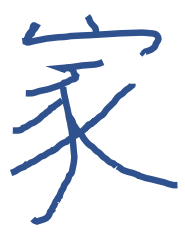

Figure 1. (A) How to write the name of Ra, the Sun God? In the upper left corner is a mouth, which corresponds to the sound "r"; under the mouth is an arm for the sound "a". Alongside them, the sun stands for ... the sun: "Ra the Sun". (B) The Chinese ideogram "jia", meaning home, reflects the transformation from an ancient pictorial word representing a house with an animal living inside.

One example is the work of Baker et al. [1] in which the progression of a neoplastic cell along a vessel can actually be seen.

Time-lapse videos are a typical application for the study of dynamic cellular and molecular activities over time. Time-lapse microscopy, in combination with other methods, is now providing a broader range of analyses. For instance, the combination of time-lapse microscopy with quantitative methods was used to investigate altered gene expression in single cells [2].

In order to report timely research progression from all cancer-related fields with the highest quality and accuracy,

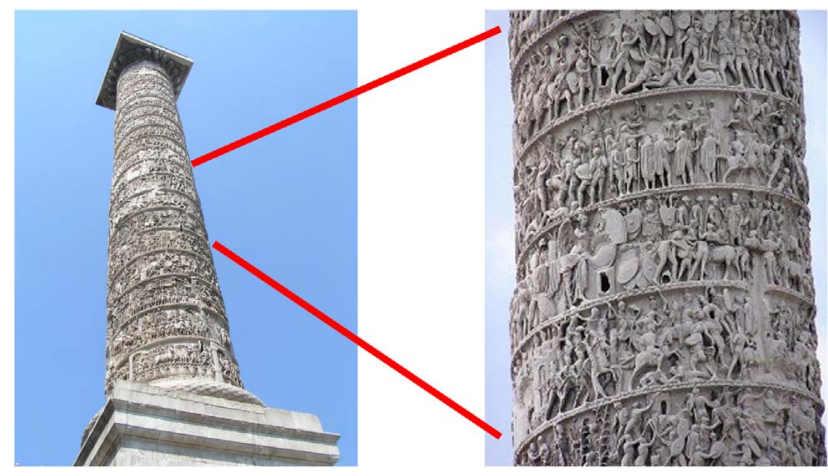

Figure 2. The Trajan's column built between 107 and 113 Anno Domini illustrates in chronological order the Roman conquest of Dacia.

thereby maximally promoting cancer research, we proudly launch Visualized Cancer Medicine as an international publication platform covering all relevant research in which moving images play a crucial role.

Accordingly, in this journal, or platform preferably named, a video is required for original research articles, review articles, clinical case studies, laboratory-based methods, surgical techniques, and a special presentation category: "Just movies".

The demand and tendency to use videos in publication are immense. We are honored to serve our authors and viewers by developing this timely and specific journal, organized by the Society of Tumor Microenvironment, China Anti-Cancer Association. Broad and strong support from the authors and readers of this journal will be of great value to the success of Visualized Cancer Medicine.

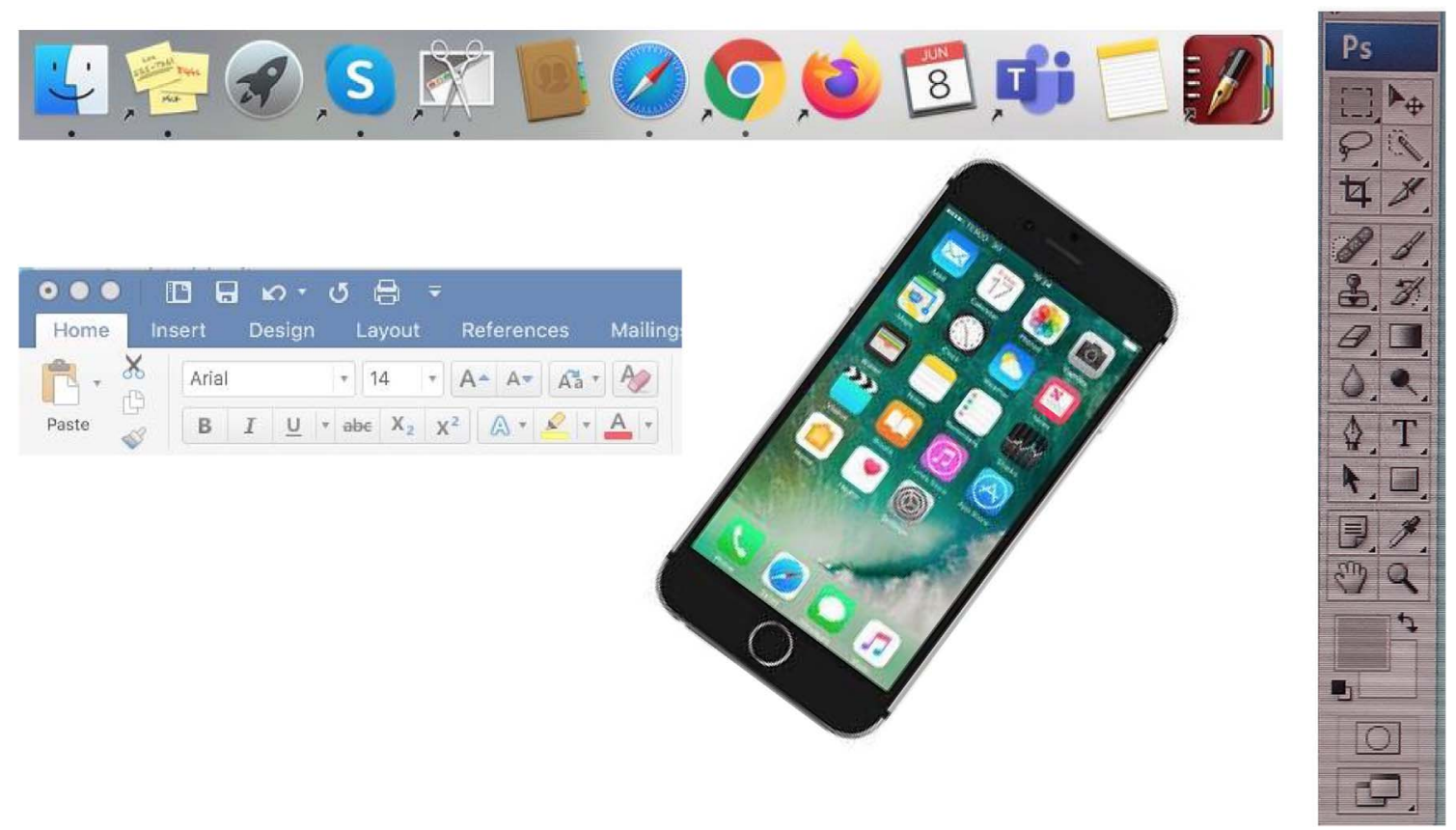

Figure 3. Screenshots from computers and smartphones: a great deal of instructions are provided by pictorial symbols instead of words. 


\section{References}

1. Baker GJ, Yadav VN, Motsch S, Koschmann C, Calinescu AA, Mineharu Y, et al. Mechanisms of glioma formation: iterative perivascular glioma growth and invasion leads to tumor progression, VEGF-independent vascularization, and resistance to antiangiogenic therapy. Neoplasia. 2014;16(7):543-561.

2. Locke J, Elowitz M. Using movies to analyse gene circuit dynamics in single cells. Nature Review Microbiology. 2009; 7(5):383-392.

Cite this article as: Qian C-N, Pezzella F \& Lu Z. Foreword for Visualized Cancer Medicine: The era for dynamic visuals is here. Visualized Cancer Medicine. 2020; 1, E1. 